\title{
Diagnostic value of tumor biomarkers CA125 and CA72-4 in differentiation of epithelial ovarian cancer and endometrioma.
}

\author{
Jafari Shobeiri Mehri', Sepasi Farnaz ${ }^{1 *}$, Dastranj Tabrizi Ali ${ }^{1}$, Mostafa Gharabaghi Parvin ${ }^{1}$, \\ Ouladsahebmadarek Elaheh ${ }^{1}$, Parizad Marziyeh ${ }^{1}$, Hakimi Parvin ${ }^{1}$, Sarbakhsh Parvin ${ }^{2}$, Ziaee \\ Mojtaba $^{3,4}$ \\ ${ }^{1}$ Women's Reproductive Health Research Center, Tabriz University of Medical Sciences, Tabriz, Iran \\ ${ }^{2}$ Department of Statistics and Epidemiology, Tabriz University of Medical Sciences, Tabriz, Iran \\ ${ }^{3}$ Phytopharmacology Research Center, Maragheh University of Medical Sciences, Maragheh, IR Iran \\ ${ }^{4}$ Cardiovascular Research Center, Tabriz University of Medical Sciences, Tabriz, Iran
}

\begin{abstract}
Ovarian cancer is the leading cause of gynecological malignancy-related mortality. CA125 level in patients with Epithelial Ovarian Cancer (EOC) and endometrioma can frequently be high. CA72-4 level was also elevated in several carcinomas including ovarian cancer. Due to its lack of specificity, however, this marker has a limited role in the differential diagnosis between EOC and endometrioma. In this research, we studied the levels of biomarkers CA125 and CA72-4 to determine their potential role in the differential diagnosis of endometrioma and EOC. For this goal, biomarkers CA125 and CA72-4 were identified in 75 patients with ovarian cancer and 75 patients with endometrioma. Cut-off levels for CA125 and CA72-4 were considered $35 \mathrm{U} / \mathrm{ml}$ and $3.8 \mathrm{U} / \mathrm{ml}$, respectively. The data have been analyzed by SPSS, with a p-value $<0.05$ considered significant. Both markers CA125 and CA72-4 were remarkably elevated in EOC $(p=0.001)$. Areas under the ROC curve for CA125 and CA72-4, with sensitivity of 0.48 and specificity of 0.52 , were 0.633 and 0.624 , respectively. The differences were not significant. It has been shown that CA125 and CA72-4 serum levels are elevated in patients with EOC; however, this did not lead to detection of malignancy. This means that CA72-4 level does not demonstrate a significant difference in malignancy diagnoses when compared to the use of CA125 alone.
\end{abstract}

Keywords: CA125, CA72-4, Epithelial ovarian cancer, Endometrioma.

Accepted on March 01, 2018

\section{Introduction}

Ovarian cancer is the seventh-most prevalent cancer worldwide and comprises about $35 \%$ of reproductive system malignancies in women [1]. Today, it represents the fifth-most common reason of deaths caused by malignancies among women. The majority of women who suffer from ovarian cancer have unspecific and enigmatic signs which lead to late diagnosis at progressed stages of the cancer. The chance of survival for this type of cancer is less than $20 \%$ after the $5^{\text {th }} y$. Practically, less than $25 \%$ of ovarian tumors are diagnosed in the early stages of the disease, while most patients have metastatic symptoms [2]. To ameliorate the prognosis and diagnose ovarian cancer in the early stages, new and effective techniques are needed to be developed.

A large number of women with ovarian cysts and pelvic masses undergo surgery. A recent study has shown that patients diagnosed with ovarian tumors have a high chance of survival upon surgical operation and tumor removal [3]. It is very important to evaluate the risk of ovarian cancer by pelvic mass assessment and by referring patients to the suitable health care centres [4]. Today, pre-symptomatically, the CA125 tumor marker is used to diagnose ovarian cancer [5]. This marker appears in $80 \%$ of progressed ovarian cancers $(>35 \mathrm{U} / \mathrm{ml})$ comparing with $50 \%$ of stage I epithelial cancers [6,7]. This means that the serum level of CA125 rises abnormally in nonovarian cancers such as endometrial, pancreas, lung, breast and colorectal cancers. Therefore, this marker has diminished specificity and cannot be used in diagnosis of endometrial cancer [5]. Moreover, CA125 levels are generally higher under the condition of benign non-gynecological diseases; such as tuberculosis, liver cirrhosis, pregnancy or different phases of menstruation [8]. Several studies have shown that endometrioma increases CA125 levels, but due to its low specificity in differentiation, clinically it has a low value [9].

CA72-4 is another biomarker that rises in response to ovarian adenocarcinoma, stomach cancer, colon cancer and breast cancer [10]. This marker can be simultaneously evaluated 
along other biomarkers; such as CA125. Notably, CA72-4 levels do not increase in response to pregnancy and menstruation phases, thus it is worthwhile to trace these tumor markers in patients suffering from ovarian epithelial tumors [6,11]. Recent studies have shown that CA125 and CA72-4 markers, when used together, demonstrate high specificity in diagnosis of ovarian cancers and high levels of these factors synchronically, or CA72-4 singularly, provide the highest accuracy rate in diagnosis of the disease $[12,13]$.

In patients with ovarian endometrioma, surgical treatment results in adverse effects in the form of destruction of the ovarian tissue and loss of egg resources. Unfortunately, this type of surgery is unavoidable in patients who are under ovarian stimulatory treatments for infertility or those that are suspected for ovarian epithelial malignancies. In women with high serum level of CA72-4, this marker can discriminate ovarian epithelial cancer (13). Specificity of CA72-4 (90\%) in ovarian cancer was significantly higher than CA125 (67\%). However, in endometerioma, the specificity of CA125 (56.1\%) was higher than that of CA72-4 (7\%) [11]. Regarding the importance of differentiation between endometrioma and ovarian cancer, this study evaluated the diagnostic values of two tumor markers CA125 and CA72-4.

\section{Materials and Methods}

\section{Study population}

This prospective study was conducted from June 2014 to May 2016 in the oncology and surgery section of the Alzahra Educational Hospital of Tabriz, with permission from the Ethics Committee of Tabriz University of Medical Sciences code no 935 in accordance with the principles outlined in the Declaration of Helsinki. 150 women diagnosed with an ovarian cyst or pelvic mass through sonography (scheduled to undergo surgery for mass removal) were eligible for enrolment in the study. Exclusion criteria included chronic disease, history of any cancer and previous chemotherapy, current hormonal therapy and pregnancy. Seventy five patients signed with ovarian cancer through clinical and laboratory data were placed in the malignant group, while the other half, who was diagnosed with benign tumors, was selected for the benign group. Patients diagnosed with ovarian cancer were differentiated based on the Federation of Obstetrics and Gynecology Guidelines (FIGO); however the type, stage and grade of tumor were determined later. All patients signed the consent declaration form [14].

\section{Laboratory analysis}

$5 \mathrm{ml}$ of peripheral blood samples were drawn from all and within an hour were centrifuged at $3000 \mathrm{rpm}$ for $10 \mathrm{~min}$ at $4{ }^{\circ} \mathrm{C}$. Thereafter, blood samples were dispensed into $5 \mathrm{~cm}^{3}$ cryotubes and were kept at $-80^{\circ} \mathrm{C}$.

CA125 level was analyzed using commercial chemiluminescent enzyme immunoassay (MyBioSource Co, San Diego, USA).
Serum biomarker level was measured for CA72-4 using ELISA kit (IBL, Hamburg, Germany). All assays were carried out by personnel who had no knowledge on t clinical history of the patients. Additionally, all surgical samples were processed for pathological examination by a gynecologic pathologist, and each diagnosis was reviewed and classified as either benign or malignant.

\section{Statistical analysis}

Statistical analysis was performed using SPSS, version 21.0 (SPSS Inc., Chicago, IL). Normal distribution of variables was defined by the Kolmogorov-Smirnov test. The results of continuous normally distributed variables were shown as the mean \pm standard deviation (SD). Nonparametric statistical tests, such as the Mann-Whitney U test or Pearson's chi-square test, were utilized to evaluate the statistical significance of differences between groups when appropriate. Normally distributed continuous variables were compared using Student's t test and non-normally distributed variables, analyzed by the Mann-Whitney U test. ANOVA was applied for other clinical parameters. $p$ values of less than 0.05 were considered statistically significant.

\section{Results}

The mean age of patients was $40.5 \pm 13.7$ y. 75 women diagnosed with ovarian endometrioma (mean age: $33.6 \pm 8.1 \mathrm{y}$, range: 16-58) were classified as the benign ovarian tumor group, and the remaining 75 patients with ovarian carcinoma were parted as the malignant group (mean age: $47.3 \pm 14.6 \mathrm{y}$ old, range: 24-79). Histology confirmed the diagnosis, and staging was conducted according to FIGO [14]. Serous adenocarcinoma was found the most common malignant tumor type of the ovary (53\%), whereas patients diagnosed with stage $3(65.3 \%)$ and grade $3(69.3 \%)$ were more frequent.

CA125 and CA72-4 serum biomarker levels were assessed in both groups. In patients from the benign group, the average CA125 level was $131.0 \pm 242.0 \mathrm{U} / \mathrm{mL}$, while in other group, the average level was $255.3 \pm 349.4 \mathrm{U} / \mathrm{mL}$. The mean difference in CA125 levels between both benign and malignant groups was statistically significant $(\mathrm{p}=0.005)$.

For the benign group, the average CA72-4 level was $2.6 \pm 6.5$ $\mathrm{U} / \mathrm{mL}$, while in the malignant group; the average level for this biomarker was $3.5 \pm 5.5 \mathrm{U} / \mathrm{mL}$. The difference in CA72-4 levels between both groups was statistically significant $(\mathrm{p}=0.003)$.

Table 1. Frequency distribution of patients with malignant ovarian tumor; histopathology, tumor grade and stage $(n=75)$.

\begin{tabular}{lll}
\hline Histology & Frequency & Percentage \\
\hline Serous cyst adenocarcinoma & 40 & 0.53 \\
\hline Mucinous cystadenoma & 17 & 0.23 \\
\hline Endometrioid adenocarcinoma & 12 & 0.16 \\
\hline Clear cell adenocarcinoma & 6 & 0.08 \\
\hline
\end{tabular}




\section{endometrioma}

\begin{tabular}{lcl}
\hline Stage FIGO & & \\
\hline I & 3 & 0.04 \\
\hline II & 18 & 0.24 \\
\hline III & 49 & 0.654 \\
\hline IV & 5 & 0.0666 \\
\hline Tumor grade & & \\
\hline 1 & 9 & 0.12 \\
\hline 2 & 14 & 0.186 \\
\hline 3 & 52 & 0.693 \\
\hline
\end{tabular}

Table 1 demonstrates the values of CA125 and CA72-4 in patients with malignant tumors based on their different stages and grades. The difference between the average level of CA125 in patients in stages I-II (early stages) of ovarian cancer in comparison to the average level of CA125 in patients in stage III-IVs (advanced stages) was not statistically significant $(\mathrm{p}=0.763)$. The average CA72-4 level in patients in stage I-II of ovarian cancer was not statistically significant $(\mathrm{p}=0.176)$. The mean serum levels of CA125 and CA72-4 in the malignant group with grades of 1and 2 were not statistically significant in comparison to patients with grade 3 ovarian cancer (Table 2).

Table 2. Serum level of CA72-4 and CA125 of malignant ovarian tumor in various stages and grades $(n=75)$.

\begin{tabular}{lll}
\hline & CA 72-4 (U/mL) & CA 125 (U/mL) \\
\cline { 2 - 3 } & Mean \pm SD & Mean \pm SD \\
\hline Stage FIGO & \\
\hline I+II & $6.6 \pm 4.27$ & $397.7 \pm 297.3$ \\
\hline III+IV & $5.1 \pm 3.25$ & $331 \pm 239.05$ \\
\hline p value & 0.176 & 0.763 \\
\hline Tumor grade & & \\
\hline $1+2$ & $7.6 \pm 5.02$ & $212 \pm 182.2$ \\
\hline 3 & $4.3 \pm 2.9$ & $392 \pm 287.7$ \\
\hline$p$ value & 0.572 & 0.895 \\
\hline
\end{tabular}

The mean level of CA125 in benign ovarian masses was 131.0 $\pm 242.0 \mathrm{U} / \mathrm{mL}$, whereas the mean level of CA125 in malignant epithelial tumors was $255.3 \pm 349.4 \mathrm{U} / \mathrm{mL}$, the latter of which was statistically significant $(\mathrm{p}=0.005)$. The mean level of CA72-4 in benign ovarian masses was $2.69 \pm 6.5 \mathrm{U} / \mathrm{mL}$, whereas the mean level of CA72-4 in malignant epithelial tumors was $3.5 \pm 5.5 \mathrm{U} / \mathrm{mL}$, the latter of which was statistically significant $(\mathrm{p}=0.003)$.

In patients with benign ovarian masses, the mean CA125 level in menopausal women was $292.6 \pm 398.1 \mathrm{U} / \mathrm{mL}$, while in nonmenopausal women it was $234.9 \pm 322.7 \mathrm{U} / \mathrm{mL}$. Considering $\mathrm{p}=0.68$, there were no meaningful statistical differences between the two groups of menopausal and non-menopausal women.
The mean CA72-4 in menopausal women was $4.1 \pm 4.9 \mathrm{U} / \mathrm{mL}$, and in non-menopausal women it was $3.15 \pm 5.9 \mathrm{U} / \mathrm{mL}$. Considering $\mathrm{p}=0.19$, there were no meaningful statistical differences between the two groups

Finally, diagnostic performance of the discriminating biomarkers between malignant and benign ovarian tumors was assessed using ROC analysis. The resulting accuracy (ROC Area) values for CA125 and CA72-4 and their corresponding ROC curves are shown in Figure 1. The AUC of these methods was calculated, and no significant differences were observed (Figure 1).

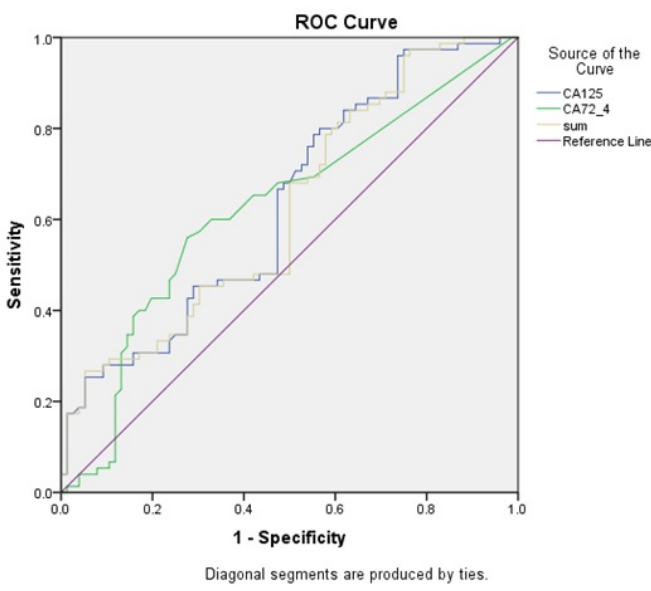

Figure 1. ROC curve for evaluation of two tumor makers in the diagnosis of ovarian malignancy.

Based on ROC analysis, if 63.3 is considered as the cut-off value for CA125, the sensitivity is equal to $48 \%$ and the specificity is equal to $52 \%$. Regarding CA72-4, when 62.4 is considered to be the cut-off value; the sensitivity level is calculated $48 \%$ and the specificity level as $50 \%$. The combination of CA125 and CA72-4 had a cut-off value at $62.5 \%$ (Figure 1). Thus, the inclusion of additional markers did not significantly increase the sensitivity. Analysis of the area under the ROC curve showed that there were no statistically significant differences in sensitivity, when CA125 and CA72-4 were combined.

\section{Discussion}

Endometriosis is a health and fertility threat that often occurs in adolescent females, even before the onset of menstruation. Several studies reported delay in diagnosis of endomerioma and EOC [15]. There are reasons to believe that delay in diagnosis of endomerioma and EOC may cause serious damage in young women and endanger their normal life and fertility as a major global public health problem [16,17]. The differential diagnosis of epithelial ovarian cancer and endometrioma is crucial and non-specific symptoms may result in serious problems for the patient. The current diagnosis of endometrioma is based on the clinical and imaging technique evaluations which are confirmed by surgery along with histological examination. Although, CA125 is applied in diagnostic and prognostic approaches, but this marker has a 
limited specificity to differentiate between endometriosis and ovarian cancer [13]. Recently, many attempts were made to find new tumor markers to develop a simple blood test for early detection of epithelial ovarian malignancies, however failed to conquer the high mortality rate of the disease. Combinations of multi- tumor biomarkers have been proven to amplify the sensitivity of the early stages diagnosis in comparison to that of a single type [18].

In this study, we monitored the CA125 and CA72-4 levels in ovarian masses of 75 benign patients versus 75 malignant subjects.

Confirming previous studies, CA125 level was elevated in $80 \%$ of patients with epithelial ovarian cancers, but in only half of them at the early stages of the disease [13,19-22]. Unfortunately, the sensitivity and specificity of CA125 in detection of the early stages of cancer were too low to have clinical value [23-25].

CA125 has also been examined to predict the presence of an ovarian malignancy in women diagnosed with a pelvic mass. For example, O'Connell et al. showed that a CA125 level above $65 \mathrm{U} / \mathrm{ml}$ in postmenopausal women with a pelvic mass had a sensitivity level of $98 \%$ in predicting the presence of an ovarian malignancy [26]. Likewise, other studies have shown that premenopausal patients with serum CA125 levels between $35 \mathrm{U} / \mathrm{ml}$ and $65 \mathrm{U} / \mathrm{ml}$ have a $50 \%$ to $60 \%$ risk of ovarian cancer $[27,28]$. The sensitivity and specificity levels of any single or multiple serum biomarkers would need to be significantly higher than that achieved with serum CA125 alone in order to be useful as a triage test before surgery. Thus, our results are in concordance with previous studies which reported that CA 125 is not a trustworthy marker in the differential diagnosis between endometrioma and epithelial ovarian cancer $[5,13]$.

In addition, we demonstrated that CA125 and CA72-4, alone or in combination, do not have sufficient ability to discriminate malignant ovarian tumors in the early or advanced stages. Also, we found that both markers have been elevated considerably in the epithelial ovarian cancers in comparison with endometrioma, and both markers showed a similar statue of sensitivity. These findings contrast the study of Anastasi et al., who found that CA72-4 is an effective factor in distinguishing between a malignancy and endometriosis $[11,13]$.

In 2008, Moore and colleagues calculated AUC for several biomarkers and their combinations. They demonstrated that the prognostic value for $\mathrm{CA} 125=82.5 \%$ and for $\mathrm{CA} 72-4=77.5 \%$, with a 95\% confidence level [5]. They suggested that the combination of HE4+CA125+CA72-4 could distinguish benign cysts from malignancies, with a $95 \%$ confidence level [5]. The prognostic value of CA125+CA72-4 was mascaraed $62.7 \%$, with $90 \%$ specificity. We found a prognostic value of $62.5 \%$ for the CA125+CA72-4, which is consistent with Moore's study [5].

\section{Conclusion}

Our findings confirm that CA125 and CA72-4 are not sufficient to differentiate between epithelial ovarian cancer and endometrioma, and additional markers such as HE4 may improve the level of sensitivity. Moreover, pelvic imaging similar to that of the risk assessment of malignancy indexes would be useful for the triage of patients with benign pelvic mass or ovarian cancer.

\section{Acknowledgments}

The authors gratefully acknowledge all the health personnel and patients who participated in the study, in addition to the data collectors, supervisors and administrative staff of Alzahra Hospital, Tabriz, Iran. Special thanks to Research Vice Chancellor Tabriz University of Medical Sciences for all the material and financial support.

\section{Financial Disclosure}

There is no financial disclosure.

\section{Funding Support}

This article was supported by Research Vice Chancellor of Tabriz University of Medical Sciences, Tabriz, Iran.

\section{References}

1. Weiderpass E, Labrèche F. Malignant tumors of the female reproductive system. Safety and Health at Work 2012; 3: 166-180.

2. Prat J. Staging classification for cancer of the ovary, fallopian tube, and peritoneum. Int J Gynecol Obstetr 2014; 124: $1-5$.

3. Jafari-Shobeiri M, Parizad M, Nazari F, Ouladsahebmadarek E, Sayyah-Melli M, MostafaGharabaghi P. Diagnostic value of HE4, CA125 and risk of ovarian malignancy algorithm in detecting ovarian cancer. Int J Women's Health Reprod Sci 2015; 3: 208-211.

4. Nossov V, Amneus M, Su F, Lang J, Janco JMT, Reddy ST. The early detection of ovarian cancer: from traditional methods to proteomics. Can we really do better than serum CA-125? Am J Obstetr Gynecol 2008; 199: 215-223.

5. Moore RG, Brown AK, Miller MC, Skates S, Allard WJ, Verch T. The use of multiple novel tumor biomarkers for the detection of ovarian carcinoma in patients with a pelvic mass. Gynecol Oncol 2008; 108: 402-408.

6. Kristjansdottir B, Levan K, Partheen K, Sundfeldt K. Diagnostic performance of the biomarkers HE4 and CA125 in type I and type II epithelial ovarian cancer. Gynecol Oncol 2013; 131: 52-58.

7. Mohammed RE, Sid Ahmed RM, Alhaj AM. The application of CA 125 in the diagnosis and follow up of ovarian cancer. Oncology 2016.

8. Anderson GL, McIntosh M, Wu L, Barnett M, Goodman G, Thorpe JD. Assessing lead time of selected ovarian cancer 
biomarkers: a nested case-control study. J Nat Cancer Institute 2010; 102: 26-38.

9. Marcus CS, Maxwell GL, Darcy KM, Hamilton CA, McGuire WP. Current approaches and challenges in managing and monitoring treatment response in ovarian cancer. J Cancer 2014; 5: 25-30.

10. Duffy M, Lamerz R, Haglund C, Nicolini A, Kalousová M, Holubec L. Tumor markers in colorectal cancer, gastric cancer and gastrointestinal stromal cancers: European group on tumor markers 2014 guidelines update. Int J Cancer 2014; 134: 2513-2522.

11. Anastasi E, Granato T, Falzarano R, Storelli P, Ticino A, Frati L. The use of HE4, CA125 and CA72-4 biomarkers for differential diagnosis between ovarian endometrioma and epithelial ovarian cancer. J Ovarian Res 2013; 6: 44.

12. Jayson GC, Kohn EC, Kitchener HC, Ledermann JA. Ovarian cancer. The Lancet 2014; 384: 1376-1388.

13. Anastasi E, Manganaro L, Granato T, Benedetti Panici P, Frati L, Porpora MG. Is CA72-4 a useful biomarker in differential diagnosis between ovarian endometrioma and epithelial ovarian cancer? Dis Markers 2013; 35: 331-335.

14. Moore RG, McMeekin DS, Brown AK, DiSilvestro P, Miller MC, Allard WJ. A novel multiple marker bioassay utilizing HE4 and CA125 for the prediction of ovarian cancer in patients with a pelvic mass. Gynecol Oncol 2009; 112: 40-46.

15. Laufer M, Goitein L, Bush M, Cramer D, Emans S. Prevalence of endometriosis in adolescent girls with chronic pelvic pain not responding to conventional therapy. J Pediatr Adolesc Gynecol 1997; 10: 199-202.

16. Brosens I, Puttemans P, Gordts S, Campo R, Gordts S, Benagiano G. Early stage management of ovarian endometrioma to prevent infertility. Facts, Views Vision in Obgyn 2013; 5: 309.

17. Khorrami A, Ghanbarzadeh S, Ziaee M, Arami S, Vajdi R, Garjani A. Dietary cholesterol and oxidised cholesterol: effects on sperm characteristics, antioxidant status and hormonal profile in rats. Andrologia 2015; 47: 310-317.

18. Muinao T, DekaBoruah H, Pal M. Diagnostic and prognostic biomarkers in ovarian cancer and the potential roles of cancer stem cells-an updated review. Exp Cell Res 2017.

19. Bast Jr RC, Klug TL, John ES, Jenison E, Niloff JM, Lazarus H. A radioimmunoassay using a monoclonal antibody to monitor the course of epithelial ovarian cancer. New Engl J Med 1983; 309: 883-887.

20. Zurawski VR, Knapp RC, Einhorn N, Kenemans P, Mortel $\mathrm{R}$, Ohmi K. An initial analysis of preoperative serum CA
125 levels in patients with early stage ovarian carcinoma. Gynecol Oncol 1988; 30: 7-14.

21. Moore RG, Miller MC, Steinhoff MM, Skates SJ, Lu KH, Lambert-Messerlian G. Serum HE4 levels are less frequently elevated than CA125 in women with benign gynecologic disorders. Am J Obstetr Gynecol 2012; 206: 351 .

22. Kurdoglu Z, Gursoy R, Kurdoglu M, Erdem M, Erdem O, Erdem A. Comparison of the clinical value of CA 19-9 versus CA 125 for the diagnosis of endometriosis. Fertility Sterility. 2009; 92: 1761-1763.

23. Campbell S, Royston P, Bhan V, Whitehead M, Collins W. Novel screening strategies for early ovarian cancer by transabdominal ultrasonography.Int J Obstetr Gynaecol 1990; 97: 304-311.

24. Jacobs I, Davies AP, Bridges J, Stabile I, Fay T, Lower A. Prevalence screening for ovarian cancer in postmenopausal women by CA 125 measurement and ultrasonography. BMJ 1993; 306: 1030-1034.

25. DePriest P, Van Nagell J, Gallion H, Shenson D, Hunter J, Andrews S. Ovarian cancer screening in asymptomatic postmenopausal women. Gynecol Oncol 1993; 51: 205-209.

26. O'connell GJ, Ryan E, Murphy KJ, Prefontaine M. Predictive value of CA 125 for ovarian carcinoma in patients presenting with pelvic masses. Obstetr Gynecol 1987; 70: 930-931.

27. Einhorn N, SjÖvall K, Knapp RC, Hall P, Scully RE, BAST Jr RC. Prospective evaluation of serum CA 125 levels for early detection of ovarian cancer. Obstetr Gynecol 1992; 80: 14-18.

28. Di-Xia C, Schwartz PE, Xinguo L, Zhan Y. Evaluation of CA 125 levels in differentiating malignant from benign tumors in patients with pelvic masses. Obstetr Gynecol 1988; 72: 23-27.

\section{*Correspondence to}

Sepasi Farnaz

Women's Reproductive Health Research Center

Tabriz University of Medical Sciences

Tabriz

Iran 